\title{
Neumopatía por humo de leña Un estudio en autopsias
}

\author{
Diana M. Palacios, Odilio Méndez
}

\begin{abstract}
Resumen
Se revisaron 1.839 protocolos de autopsia del hospital San Juan de Dios de Santa Fe de Bogotá 155 pacientes tenían exposición al humo de los cuales $103(5,6 \%)$ reunían los criterios de inclusión. $97 \%$ de los casos eran mujeres. Los cambios histológicos principales fueron: enfermedad vascular hipertensiva (39\%), tromboembolismo pulmonar de vasos de pequeño y mediano calibre (38\%) y antracosis peribronquial, perivascular e intersticial. En todos los parámetros histológicos se apreció mayor compromiso pulmonar con el mayor tiempo de exposición al humo de leña. A pesar de sus características peculiares no es posible clasificarla como una entidad nosológica, estando en la categotía de las neumoconiosis. En conclusión, es una condición clínico-patológica frecuente en nuestro medio, especialmente entre mujeres que han estado expuestas por muchos años al humo de leña; entre los cambios histológicos no patognomónicos, se encuentra el depósito de pigmento de antracosis, la enfermedad vascular hipertensiva, la fibrosis pulmonar y los cambios de bronquitis crónica.
\end{abstract}

\section{Pneumopathy due to inhaling firewood smoke. Autopsy findings from the San Juan de Dios Hospital}

1,839 autopsy protocols from the San Juan de Dios Hospitalwere revised, 155 patients had been exposed to smoke, of whom $103(5.6 \%)$ met the inclusion criteria. 97\% were women. The principal histological changes were: hypertensive vascular disease (39\%), pulmonary thrombo-embolism of the small and medium calibre vessels $(38 \%)$ and peribronchial, perivascular and interstitial anthracosis. In all histological parameters, greater pulmonary compromise was seen in relation to greater exposure to firewood smoke. In spite of these peculiar characteristics, it is not possible to classify it as a nosological entity, being in the pneumoconiosis category. In conclusion it is a frequently occuring clinical-pathological condition in our environment, especially amongst women who have been exposed to firewood smoke for many years. Amongst the non-pathognomic histological changes, anthracosis pigment deposits, hypertensive vascular disease, pulmonary fibrosis and chronic bronchitis changes were found.

La neumopatía por humo de leña es una enfermedad pulmonar asociada con una exposición intensa y prolongada al humo de leña en el interior de la casa. Se han observado por varios

Departamento de Patología, Hospital San Juan de Dios, Universidad Nacional de Colombia, Santa Fe de Bogotá, Colombia.

Recibido para su publicación: 17 de febrero de 1998. - Aceptado para su publicación: 11 de mayo de 1998. 
años pacientes, especialmente mujeres, con enfermedad pulmonar crónica, hipertensión arterial pulmonar severa y cardiopulmonar cuya etiología no se ha aclarado com-pletamente. Todos tenían el antecedente de exposición prolongada al humo de leña (1-4). Se ha demostrado que el humo de leña contiene una serie de partículas irritantes importantes como sílice, fósforo, hierro, azufre, carbón y ceniza; gases como el monózido de carbono, óxido de sulfuro, óxido nitroso, aldehídos y material poliorgánico como hidrocarburos aromáticos policíclios (5). En una revisión realizada por Pryor, compara la química del humo de cigarrillo, humo de leña, humo de plásticos y materiales de construcción que inhalan personas atrapadas en incendios. La exposición aguda al humo en un incendio produce pérdida de la función pulmonar. La inhalación de humo de cigarrillo y de humo de leña tiene similitudes con las del humo inhalado en un incendio: altas concentraciones de monóxido de carbono, productos de combustión $y$ radicales libres (6).

La neumoconiosis es la enfermedad resultante de la acumulación de diferentes tipos de polvos en el pulmón. Las neumoconiosis más comunes inductoras de fibrosis pulmonar son la silicosis, la neumopatía de los trabajadores del carbón y la asbestosis (7-10). Además de las anteriomente descritas, también induce enfermedad pulmonar la exposición a otros materiales inorgánicos como la antracosis, la beriliosis y la talcosis, entre otras $(9,11)$. Aún no existe acuerdo entre los expertos si la es una neumoconiosis separada o, si como opina la mayoría, es una variedad de silicoantracosis (3, 9). Estudios hechos en Nepal, Méjico y Colombia evidenciaron la presencia de síntomas respiratorios que, en etapas iniciales, afectaban a mujeres expuestas por largos años, especialmente en recinto mal ventilado y se caracterizaban por tos productiva y disnea. Generalmente, las pacientes no han sido fumadoras, provienen de áreas frías y no presentan patología pulmonar o cardiaca previa. La edad de presentación suele ser después de la quinta década de la vida y la mayoría ha estado expuesta por treinta o más años al humo de la leña, al utilizarla para cocinar en su hogar $(1,2,4,12-14)$.
Los estudios histológicos de neumopatía por humo de leña han demostrado cambios compatibles con bronquitis crónica moderada a severa, siendo los principales hallazgos los siguientes: engrosamiento de la membrana basal con fibrosis e inflitrado inflamatorio a expensas de linfocitos y células plasmáticas; hiperplasia de células caliciformes; infiltrado inflamatorio agudo y crónico de la submucosa con depósito de material antracrótico; hiperplasia de glándulas mucosas y metaplasia escamosa del epitelio bronquial. En pacientes sometidos a biopsia pulmonar a cielo abierto, la microscopía de luz evidenció engrosamiento de los tabiques alveolares por depósito de colágeno, así como infiltrado celular linfocítico con depósito antracótico como hallazgo notorio, siendo más prominente en el tejido conectivo que rodea vías aéreas pequeñas y arterias. Ocasionalmente se han evidenciado trombos antiguos recanalizados. Estos hallazgos histológicos comparten alguna similitud con los encontrados en otras formas de exposición a polvos inorgánicos con enfermedad pulmonar intersticial $(1-3,9)$. Teniendo en cuenta el efecto irritante y mutagénico sobre la vía aérea del humo de leña, parece necesario alertar sobre la posible relación causa-efecto entre este agente agresor y los casos de carcinoma escamocelular y la presencia de atipias en las células epiteliales (2).

A pesar de que por muchos años en nuestro medio ha sido notable la presencia de enfermedad pulmonar en personas expuestas por los años al humo de la leña, especialmente de sexo femenino, pocas han sido las investigaciones dirigidas a esclarecer su naturaleza y su incidencia. No existen trabajos sobre hallazgos de autopsia, lo cual nos motivó a observar los principales cambios histológicos en estudios de autopsia de pacientes del Hospital San Juan de Dios en Santa Fe de Bogotá.

\section{Materiales y métodos}

Se realizó un estudio descriptivo, retrospectivo, de casos y controles revisando todos los protocolos de autopsia del Departamento de Patología del Hospital San Juan de Dios a partir del 1 
de enero de 1983 hasta el 31 de diciembre de 1995 con diagnóstico de neumopatía por humo de leña o que tuvieran como antecedente exposición al humo de leña por más de diez años, sin exposición al cigarrillo. Se tomó como controles a pacientes con el antecedente de ser fumadores y que no hubieran estado expuestos al humo de leña.

Se llenó un formulario con datos generales como: tipo de exposición, procedencia, principales hallazgos clínicos, paraclínicos, radioló-gicos, procedimientos diagnósticos, histoló-gicos y hallazgos de autopsia.

\section{Resultados}

En el cuadro 1 se detalla el número de necrop-sias $y$ de casos por año. En total, se revisaron 1.839 protocolos, de los cuales se encontraron $155 \mathrm{con}$ historia de exposición y los 9 restantes estuvieron expuestos durante menos de 10 años. Así, pues, 103 $(5,6 \%)$ reunían los criterios de inclusión o sea, presentaron exposición por más de 10 años al humo de la leña.

En el cuadro 2 se aprecian las características epidemiológicas de ambos grupos. La edad promedio de los pacientes es similar en ambos grupos y la mayoría son pacientes de edad avanzada (promedio, 67,5 años en los casos vs. 64,2 en el grupo control). $94 \%$ de los casos eran mujeres en el grupo de casos contra $11 \%$ en el

Cuadro 1. Autopsias de neumopatía por humo de leña, HSJD, 1938-1995.

\begin{tabular}{lccc}
\hline Año & Total autopsias & Total NHL & $\%$ \\
\hline 1938 & 158 & 2 & 1,27 \\
1984 & 154 & 3 & 1,95 \\
1985 & 111 & 3 & 2,70 \\
1986 & 206 & 13 & 6,31 \\
1987 & 104 & 5 & 4,81 \\
1988 & 153 & 7 & 4,58 \\
1989 & 84 & 4 & 4,76 \\
1990 & 214 & 7 & 3,27 \\
1991 & 85 & 9 & 10,59 \\
1992 & 154 & 9 & 5,84 \\
1993 & 163 & 16 & 9,82 \\
1994 & 138 & 12 & 8,70 \\
1995 & 115 & 13 & 11,30 \\
\hline Total & 1.839 & 103 & 5,60 \\
\hline
\end{tabular}

Cuadro 2. Datos epidemiológicos.

\begin{tabular}{lrr}
\hline & $\begin{array}{c}\text { Casos } \\
103(100 \%)\end{array}$ & $\begin{array}{r}\text { Controles } \\
40(100 \%)\end{array}$ \\
\hline Edad promedio & 67,5 años & 64,2 años \\
Sexo masculino & $6(6 \%)$ & $29(72,5 \%)$ \\
sexo femenino & $97(94 \%)$ & $11(27,5 \%)$ \\
Procedencia & & \\
Cundinamarca & $53(51,5 \%)$ & $31(77,5 \%)$ \\
Boyacá & $35(34 \%)$ & $2(5 \%)$ \\
Santander & $7(6,8 \%)$ & $1(2,5 \%)$ \\
\hline
\end{tabular}

grupo control.

Los cambios histológicos más llamativos asociados a la exposición al humo de leña fueron los siguientes: enfermedad vascular hipertensiva (EVH) presente en 40 casos $(38,8 \%$ ) y en 8 controles $(20 \%), p=0,05$ y RR 3,78 (figuras 1 y 2). La EVH de grado IV a VI se apreció en $7,8 \%$ de los casos (31) que en los controles $(20 \%) p=0,15$ y RR 1,98 ; tromboembolismo pulmonar de vasos de pequeño y mediano calibre en 39 casos (38\%) y en 6 controles ( $15 \%$ ), $p=0,014$, RR 5,96. Se encontró depósito de pigmento antrácotico peribronquial, perivascular e intersticial (figuras 3 a 5) en forma estadísticamente significativa en los casos y, además, depósito antracótico subpleural aunque más frecuente $\sin$ significancia en los casos y, además, depósito antracótico subpleural aunque más frecuente $\sin$ significancia estadística, como puede apreciarse en el cuadro 3.

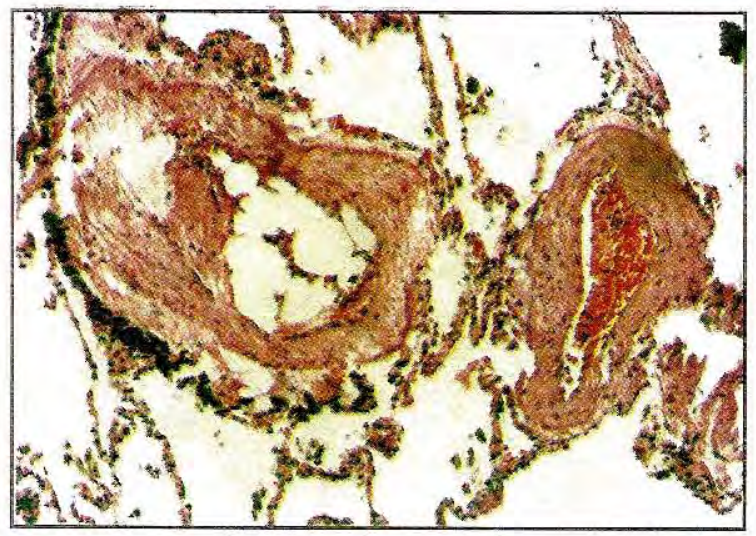

Figura 1. Cambios de enfermedad hipertensiva grado II, trombo de médula ósea, depósito de pigmento de antracosis perivascular, hematoxilina-eosina, $400 \mathrm{X}$. 


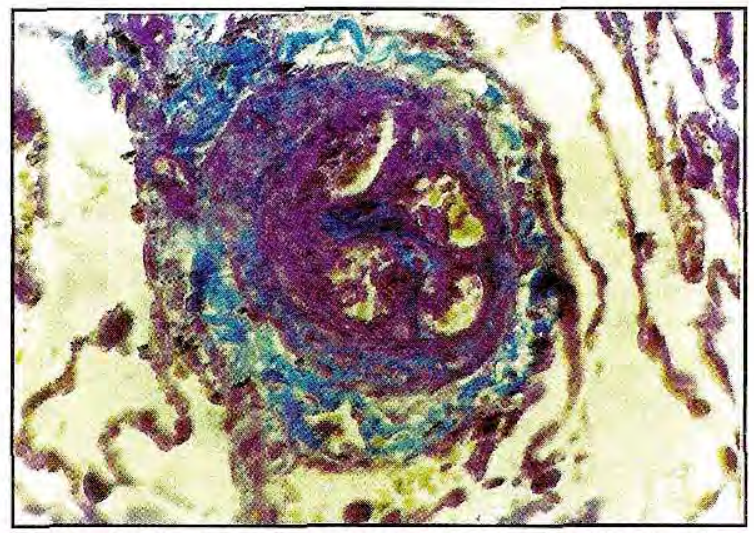

Figura 2. Cambios de enfermedad vascular hipertensiva, tricrómico de Masson, $400 \mathrm{X}$.

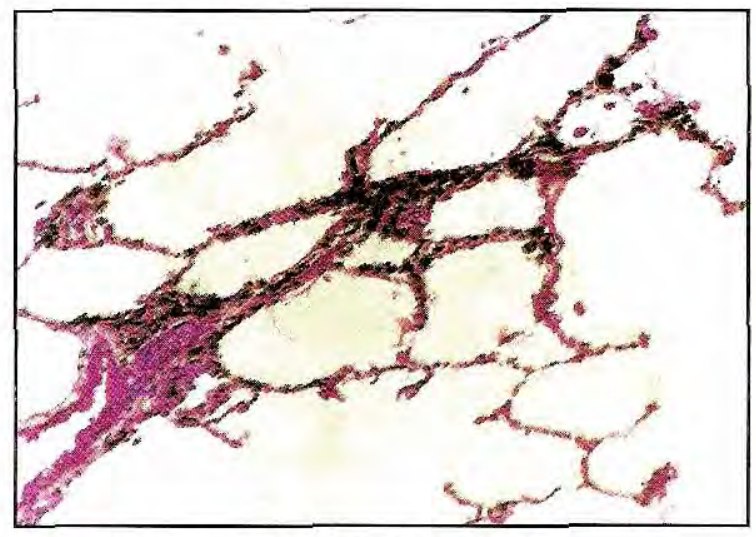

Figura 3. Antracosis intersticial, HE $400 \mathrm{X}$.

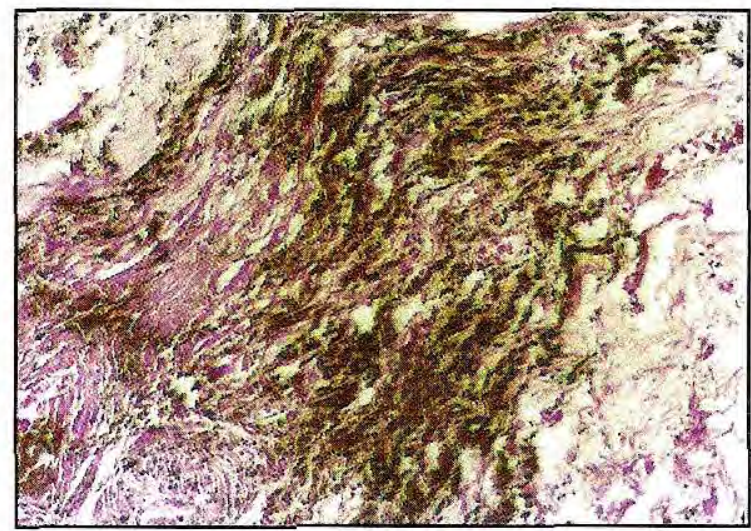

Figura 4. Fibroantracosis, HE $400 \mathrm{X}$.

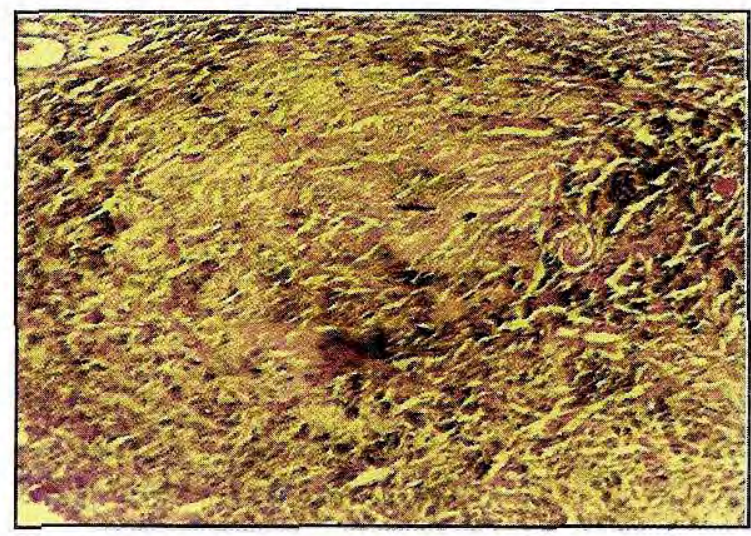

Figura 5. Nódulo silicoantracótico, HE 400 X.

Cuadro 3. Principales hallazgos histológicos, datos totales.

\begin{tabular}{lrrrrrr} 
& Casos & \% & Control & $\%$ & p & RR \\
\hline EVH, grado I-II-III & 32 & 31,0 & 8 & 20,0 & 0,26 & 1,25 \\
EVH, grado IV-V-VI & 8 & 7,8 & 0 & 0,0 & 0,15 & 1,98 \\
Total EVH & 40 & 38,8 & 8 & 20,0 & 0,05 & 3,78 \\
Tromboembolismo & 39 & 37,9 & 6 & 15,0 & 0,014 & 5,96 \\
Depósito antracótico peribronquial & 57 & 55,3 & 11 & 27,5 & 0,005 & 7,87 \\
Depósito antracótico perivascular & 57 & 55,3 & 8 & 20,0 & 0,002 & 13,10 \\
Depósito antracótico intersticia! & 65 & 63,1 & 9 & 22,5 & $0,0000217,40$ \\
Depósito antracótico subpleural & 13 & 12,6 & 1 & 2,5 & 0,12 & 2,30 \\
Metaplasia ósea & 8 & 7,8 & 2 & 5,0 & 0,8 & 0,05 \\
Neoplasia asociada & 4 & 3,9 & 2 & 5,0 & 0,86 & 0,03 \\
Bronquiectasias & 30 & 29,1 & 10 & 25,0 & 0,77 & 0,08 \\
BOoP & 5 & 4,9 & 0 & 0,0 & 0,36 & 0,83 \\
Engrosamiento MB bronquial, inflamación crónica & 7 & 6,8 & 5 & 12,5 & 0,44 & 0,59 \\
Hiperplasia de células caliciformes & 28 & 27,2 & 13 & 32,5 & 0,67 & 0,08 \\
Metaplasia escamosa del epitelio bronquial & 6 & 5,8 & 4 & 10,0 & 0,60 & 0,26 \\
\hline
\end{tabular}


más frecuente sin significancia estadística, como puede apreciarse en el cuadro 3.

Se encontraron 5 casos de bronquiolitis obliterante con neumonía organizada (BOOP) en el grupo de casos, pero, ninguno en el grupo control ( $p=0,36$, RR 0,83). En $29 \%$ de los casos se encontró bronquiectasis contra $25 \%$ controles ( $p=0,77$ y RR 0,08$)$. Ocho casos $(7,8 \%)$ contra 2 controles (5\%) tenían metaplasia ósea pulmonar intraparenquimatosa $(p<0,05)$, y 4 $(3,9 \%)$ neoplasias contra $1(2,5 \%)$ de los controles, las cuales se detallan más adelante.

Los siguientes hallazgos histológicos se encontraron con mayor frecuencia en el grupo control (fumadores) que en los expuestos al humo de leña, pero, sin significancia estadística: engrosanmiento del tabique alveolar, hiperplasia de células calciformes con índice de Raid positivo y metaplasia escamosa del epitelio bronquial (cuadro 3).

Según el grado de exposición, los pacientes se clasificaron en tres grupos, así: 10 a 25 años de exposición, 25 a 45 años y más de 45 años. En todos los parámetros histológicos mencionados en el cuadro 4, se apreció una tendencia hacia el mayor compromiso pulmonar con mayor tiempo de 45 años, respectivamente. EI segundo hallazgo más frecuente fue la presencia de ganglios antracóticos (54, 58 y

Cuadro 4. Hallazgos histológicos según tiempo de exposición.

\begin{tabular}{lccc}
\hline & $\begin{array}{c}10-25 \text { años } \\
\%\end{array}$ & $\begin{array}{c}\mathbf{2 5 - 4 5} \text { años } \\
\%\end{array}$ & $\begin{array}{c}>45 \text { años } \\
\%\end{array}$ \\
\hline Antracosis & 79 & 87 & 96 \\
Ganglio linfático & 54 & 58 & 67 \\
antracótico & & & \\
Enfermedad vascular & 33 & 29 & 48 \\
hipertensiva & 25 & 26 & 38 \\
$\quad$ grados I-III & 8 & 3 & 10 \\
$\quad$ grados IV-VI & 33 & 26 & 44 \\
Fibrosis pulmonar & 333 & 5 & 41 \\
Tromboembolismo & 21 & 29 & 33 \\
Bronquitis crónica & 21 & 29 & 33 \\
Bronquiectasas & 12 & 13 & 21 \\
Nódulos fibróticos en & & 13 & 19 \\
bulbo de cebolla & 12 & 3 & 6 \\
Necrosis pulmonar & 12 & & \\
BOOP & 4 & & \\
\hline
\end{tabular}

Cuadro 5. Patologías asociadas.

\begin{tabular}{lrrrrrr}
\hline Entidad & \multicolumn{3}{c}{ Casos } & \multicolumn{2}{c}{ Controles } & \\
& $\mathbf{n}$ & $\%$ & $\mathbf{n}$ & $\%$ & & RR \\
& 84 & 81,6 & 24 & 60,0 & 0,01 & 6,12 \\
\hline Arterioesclerosis & 57 & 55,3 & 27 & 67,5 & 0,25 & 1,29 \\
Edema pulmonar & 44 & 42,8 & 8 & 20,0 & 0,02 & 5,48 \\
Falla cardiaca & 36 & 35,0 & 14 & 35,0 & 0,84 & 0,04 \\
Bronconeumonía & 34 & 33,0 & 17 & 42,5 & 0,38 & 0,76 \\
Ulcera gástrica & 31 & 30,1 & 7 & 17,5 & 0,18 & 1,74 \\
Desnutrición & 31 & 30,1 & 7 & 17,5 & 0,18 & 1,74 \\
Hígado graso & 25 & 24,3 & 3 & 7,5 & 0,04 & 4,14 \\
TEP & 22 & 21,4 & 3 & 7,5 & 0,08 & 2,94 \\
Infarto pulmonar & 17 & 16,5 & 2 & 5,0 & 0,12 & 0,39 \\
ACV & 16 & 15,5 & 4 & 10,0 & 0,55 & 0,35 \\
Hemorragia pulmonar & 11 & 10,7 & 3 & 7,5 & 0,79 & 0,07 \\
TBC & 2 & 1,9 & 1 & 2,5 & 0,65 & 0,19 \\
Ca. bronquioloalveolar & & & & & & \\
Adenocarcinoma & & 1,0 & 0 & 0,0 & 0,62 & 0,24 \\
primario & 1 & 1,0 & 0 & 0,0 & 0,62 & 0,24 \\
Adenoma bronquial & 1 & 1,0 & 0 &
\end{tabular}

$67 \%$ ) y el tercero, la enfermedad vascular hipertensiva (33, 29 y 48\%), respectivamente.

Al hacer la correlación clínico-patológica, se encontró que, de los 103 pacientes expuestos, 88 $(86 \%)$ tenían cambios clínicos e histológicos similares a los descritos en otras series. De estos casos, únicamente en $9,1 \%$ el grupo médico tratante hizo el diagnóstico premortem. A su vez, el patólogo sólo hizo el diagnostico de neumopatía por humo de leña en 15 casos $(17 \%)$.

Se estudió la presencia de patologías sistémicas concominantes, siendo la arteriosclerosis la entidad más prevalente en el grupo de casos (84\%) y la segunda en el grupo control $(60 \%)$ donde predominó el edema pulmonar $(67,5 \%)$. En once casos, la autopsia demostró la presencia de tuberculosis de los cuales 5 no fueron diagnosticadas premortem. En el cuadro 5 , se detallan los principales hallazgos de patologías sistémicas concomitantes.

\section{Discusión}

La neumopatía por humo de leña es una entidad cada vez más reconocida no solo en nuestro medio sino a nivel mundial como causante de neumopatía crónica; predomina en mujeres de 
países en vía de desarrollo expuestas al humo de leña en recintos mal ventilados y por períodos prolongados de tiempo (1-5, 13-15). En el HSJD se empezó a hacer este diagnóstico en autopsias a partir de 1983 y, desde entonces hasta 1995, se encontraron entre 1.839 autopsias, 155 pacientes expuestos al humo de leña. La edad promedio de los pacientes fue de 67,5 años y $95 \%$ eran mujeres, lo cual está acorde con lo referido en la literatura, dado esto por el uso doméstico de la leña y el contacto más próximo de las mujeres con este producto $(1,2,4,12-14)$.

Analizando los hallazgos histológicos se evidencia que la antracosis es la alteración más prevalente. Llama la atención el depósito de pigmento de antracosis intersticial en $63 \%$ de los casos contra $22,5 \%$ en los controles con significancia estadística $(p=0,0002$ y RR de 17,4 , antracosis perivascular en $55,3 \%$ vs. $20 \%$, antracosis peribronquial en $55,3 \%$ vs. $27,5 \%$. La antracosis subpleural aunque no fue significativa también predominó en el grupo expuesto al humo de la leña, $12,6 \%$ contra $2,5 \%$. Esto se explica por la composición del humo de leña conformado por las sustancias inorgánicas como sílice, fósforo, carbón, etc., posiblemente implicados en el pigmento antracótico.

Otro hallazgo importante fue la enfermedad vascular hipertensiva presente en $38,8 \%$ de los casos contra $20 \%$ de los controles. Estudios previos hechos en nuestro país en pacientes con historia de exposición al humo de la leña han evidenciado la presencia de enfermedad vascular hipertensiva de grado variable (13). Otro cambio fue la presencia de trombos vasculares el cual fue mucho más frecuente en el grupo de casos que en el grupo de control (38\% vs. $15 \%$ con $p=0,014$ y RR 5,96); de los 37,19 estuvieron asociados a tromboembolismo pulmonar $y$ el resto a hipertensión pulmonar. La alta incidencia de trombos vasculares podría explicarse por el aumento de la viscosidad sanguínea que frecuentemente se presenta en las neumopatías crónicas; también es posible que el factor irritante crónico del humo de leña pueda influir contribuyendo a la presencia de la enfermedad vascular hipertensiva, aunque en esta última es difícil decir hasta que punto el compromiso de la vía aérea y la consiguiente hipoxemia, descrita en estos pacientes, sean los responsables de los cambios vasculares $(16,17)$.

Del estudio histológico podemos concluir que el depósito de pigmento antrácotico, la enfermedad vascular hipertensiva y la fibrosis de la pared alveolar intersticial son los cambios más frecuentes en los pacientes con neumopatía por humo de leña. Otros cambios como la presencia de metaplasia cartilaginosa, la calcificación del cartílago bronquial, las bronquietasias y la bronquiolitis obliterante con neumonía en organización que aunque ligeramente más frecuentes en el grupo de casos, no alcazaron significacia estadística.

Un hecho interesante fue el hallazgo de cuatro neoplasias pulmonares en el grupo de casos contra uno en el grupo control a pesar de que el tamaño de la muestra es pequeño y, por tanto, no se pueden sacar conclusiones al respecto, valdría la pena prestar la atención con diseños metodológicos dirigidos específicamente para corroborar la relación entre la exposición al humo de leña y la presencia de neoplasia pulmonar.

Al analizar las diferentes alteraciones con respecto al grado de exposición, se aprecia cómo la gran mayoría de cambios histológicos tiende a acentuarse con el mayor tiempo de exposición, especialmente después de los 45 años. Por ejemplo, la enfermedad vascular hipertensiva estuvo presente en $33 \%$ de los casos expuestos durante 10 a 25 años, permaneció en $29 \%$ en los expuestos durante 25 a 45 y aumentó hasta $48 \%$ en los expuestos durante más de 45 años.

La antracosis también mostró incremento en la incidencía a medida que la exposición aumentaba, pero con la diferencia de que ya luego de 10 años de estar inhalando humo de leña, $79 \%$ de nuestros casos mostraba este pigmento depositado en su vía aérea y prácticamente todos $(98 \%)$ aquellos que lo inhalaron durante 45 años o más lo presentaban.

Un dato histológico importante es la presencia de fibrosis pulmonar, la cual se evidenció en 
$33 \%$ de los pacientes del grupo de casos expuestos entre 10 a 25 años, en $26 \%$ de los expuestos durante 25 a 45 años y en $44 \%$ de aquellos pacientes que inhalaron humo de leña por más de 45 años. Es ampliamente reconocida la presencia de fibrosis pulmonar en pacientes con neumopatía por humo de leña, pero ningún estudio previo ha determinado la frecuencia de este hallazgo clínico-patológico.

Como un hecho llamativo, este diagnóstico no se realizó premortem ni tampoco en las necropsias, pues, en $90,9 \%$ en el $83 \%$ de los pacientes ni los clínicos ni los patólogos hicieron el diagnóstico, respectivamente. Es indispensable, entonces, que tanto clínicos como patólogos piensen en esta entidad y se familiaricen con sus manifestaciones clínicas e histológicas para llegar al diagnóstico preciso. Sin embargo, el hecho de que cada año a partir de 1983 se estén viendo más casos de esta entidad no quiere decir que sea una patología de reciente aparición, sino precisamente que se está reconociendo y diagnosticando cada vez más.

Vale la pena subrayar la frecuencia de casos de tuberculosis pulmonar, la cual se presentó en $11 \%$ de los casos y en $7,5 \%$ de los controles. De los 11 casos de tuberculosis presentes, en 5 el clínico no consideró este diagnóstico.

La bronquitis crónica se vio el mayor número de oportunidades en el grupo control $(30 \%)$ que en los casos (15\%), alcanzando significancia estadística ( $p=0,6$ y $R R 3,5$ ). Lo anterior fue debido muy seguramente al consumo importante de cigarrillo en el grupo control y a que no hubo un muestreo representativo que incluyera cortes histológicos del parénquima pulmonar, parahiliar y supbleural en el otro grupo.

En conclusión, la neumopatía por humo de leña es una condición clínico-patológica frecuente en nuestro medio, especialmente entre mujeres que han estado expuestas por muchos años; se presenta con unas características que, desde el punto de vista del patólogo, se diferencian de las observadas en personas fumadoras; puede ocasionar fibrosis pulmonar, como hallazgo particular de esta serie con grados de enfermedad vascular hipertensiva y con depósito de pigmento de antracosis como evento casi universal. A pesar de ser una patología antigua, solo en la última década se ha definido de forma conclusiva la capacidad del humo de leña de causar daño pulmonar; aún debe investigarse mucho más al respecto. Por ser una patología de países pobres, del Tercer Mundo, el avance en su conocimiento ha sido lento y las publicaciones al respecto han sido escasas pero cada vez se le reconoce más, hasta el punto de que la Organización Mundial de la Salud ya incluyó al humo de la leña comc elemento agresor y causante de enfermedad pulmonar.

\section{Agradecimientos}

Los autores desean agradecer al doctor Hernán Alvarado por sus valiosos conceptos y ayuda con las fotografías. A los profesores del Departamento de Patología de la Universidad Nacional de Colombia por sus opiniones, en especial, a la doctora Susana de Onatra, al doctor Orlando Ricaurte y a la doctora Elvira Castro de Pabón; finalmente, al doctor Jorge Hernán López Ramírez, docente de medicina interna de la Universidad Nacional por su apoyo en todo aspecto.

\section{Referencias}

1. Restrepo J, Reyes P, de Ochoa P, Patiño E. Neumoconiosis por inhalación del humo de leña. Acta Med Col 1983;8:191-204.

2. Sandoval J, Salas J, Martínez-Guerra M, Gómez A, Martínez O, Portales A, et al. Pulmonary arterial hypertension and cor pumonale associated with cronich domestic woodsmoke inhalation. Chest 1993; 103(1):12-20.

3. Reyes P. Neumoconiosis por inhalación de humo de leña. En: Camacho F, Páez J, Awad C, editores. Enfermedades del tórax. Bogotá: Ediciones Médicas Zambon; 1992.

4. Jones H. COPD in women in developing countries. Chest 1974;65:705.

5. Samet J, Marbury M, Spengler J. Health effects and sources of indoor pollution. Am Rev Respir Dis 1987;136:1486-1508.

6. Pryor WA. Biological effects of cigarette smoke, wood smoke, and the smoke from plastics: the use of electron spin resonance. Byodinamics Institute, Lousiana State University 1992;13(6):659-76. 
7. Born PJ, Schins R, Janssen YMW, Lenaerts L. Molecular basis for differences in susceptibility to coal workers pneumoconiosis. Toxicol 1992;6465:767-72.

8. McLoud TC. Occupational lung disease. Radiol Clin North Am 1991;29(5):931-41.

9. Restrepo J, et al. EPID. Neumoconiosis por humo de leña. En: Restrepo J, Maldonado E, editores. Neumología. Medellín: CIB; 1986.

10. Solarte I. Enfermedades pulmonares ocupacionales. Rev Colomb Neumol 1994;6(4):179-83.

11. Wright PH. Occupational lung diseases other than asthma. Postgrad Med J 1993;69:129-35.

12. Grobbelaar JP, Bateman ED. Hut lung: a domestically acquired pneumoconiosis of mixed etiology in rural women. Thorax 1991;46(5):334-40.
13. Denis R, Maidonado G, Norman S, Baena E, Castaño H, Martínez G, Vélez JR. Exposición a humo de leña y riesgo de enfermedad pulmonar obstructiva en mujeres. Chest 1996.

14. Pandey MR. Prevalence of chronic bronchitis in a rural community of the hill region of Nepal. Thorax 1984;39:331-6.

15. Cotes JE, Steel J. Respiratory defense mechanisms in work related lung disorders. Oxford: Blacwell Scientific Publications; 1987. p. 61-77.

16. Saiyed HN, et al. Non-occupational pneumoconiosis at high altitude villages in central Ladah. $\mathrm{Br} \mathrm{J}$ Ind Med 1991;48(12):825-9.

17. López JH, Giraldo H. Tromboembolismo pulmonar. En: Camacho F, Páez J, Awad C, editores. Enfermedades del tórax. Bogotá: Ediciones Médicas Zambón; 1992. 